\title{
What a waste of MRI-scans!
}

\author{
Jannick Brennum ${ }^{1}$ \\ Received: 14 December 2018 / Accepted: 19 December 2018 / Published online: 16 January 2019 \\ (C) Springer-Verlag GmbH Austria, part of Springer Nature 2019
}

The study by Gui et al. in this issue of Acta Neurochirurgica is a gold nugget that should guide the practice of all professionals treating patients with low-grade gliomas (LGG) [1]. The authors demonstrate that in a real-life setting, the standard radiological evaluation and report of MRI control scans performed to investigate whether there is tumor growth in patients with LGG is inadequate. Instead of the common 2-dimension measurement, they recommend the use of tumor segmentation with measurement of tumor volume and calculation of growth pattern by plotting changes in volume over time. Similar findings have made by others, but still is has not resulted in a change of practices in most departments. Inspired by the study by Mandonnet et al. [2], I segmented the volumes of FLAIR signal changes in 11 of my patients over time all reported as "no growth" by our neuroradiologist. For segmentation, I used Iplan3.1 (Brainlab $®$, Münich Germany) that allows for semi-automated segmentation. I plotted both tumor volume and the calculated mean tumor diameter over time as suggest by Mandonnet et al. [2]. I found that there was clear growth in 9 out of these 11 patients with growth rates varying from $0.25-16 \mathrm{~mm}$ average diameter change. Figure 1 demonstrates 3 out of these 11 patients with different patterns. From the left the first patient demonstrates no growth over a period of three and a half years and she will be continuously followed with MRI; the second demonstrates an increased growth rate after 2 years indicating the need for surgery either in the form of resection or bi-

This article is part of the Topical Collection on Tumor - Glioma

Jannick Brennum

Jannick.brennum@regionh.dk

1 Copenhagen Neurosurgery, Neuroscience Centre, Rigshospitalet, University of Copenhagen, Blegdamsvej 9 ,

2100 Copenhagen, Denmark opsy; the third patients was operated after 18 month "watchful waiting" and demonstrated $50 \%$ reduction in tumor volume on the early postoperative MRI with further reduction to $25 \%$ without further treatment, demonstrating the often seen reduction in gliotic FLAIR signal changes. It is often very difficult to discriminate between residual tumor and gliosis after surgery. Such discrimination is easier if preoperative and postoperative images are fused and compared. It is possible to decide whether FLAIR signal changes are within the previous tumor volume or in the peritumoral zone where gliotic changes are to be expected following surgery.

Having already performed the tumor segmentation for the different time points from before surgery and during follow-up, I find it additionally helpful to present overlays over the tumor volumes to identify the anatomical pattern of tumor growth (Fig. 2). In a patient with previous surgery, there will often be gliotic changes and these will often be more prominent if the patient has also received radiation therapy. It is not given that regrowth will happen in all directions, which becomes obvious when studying all segmentations overlaid; hence, this is an aid in the presurgical planning.

My personal experience aligns completely with the study presented by Gui et al. I found tracking of tumor volume over time to be easy and very helpful in identifying tumor growth and potential malignant degeneration. The visualization of 3-D growth pattern by overlying the segmentations from different time points further increases the ability to discriminate between FLAIR signal changes representing tumor and those more likely representing gliotic tissue. In my small series of 11 patients, multiple MRI-scans were wasted in 9 of the patients as tumor growth was missed by the standard evaluation with twodimensional measurements. I strongly urge the use of 3-D segmentation. This is soon likely to become even easier when sufficiently robust automated segmentation software become available. 

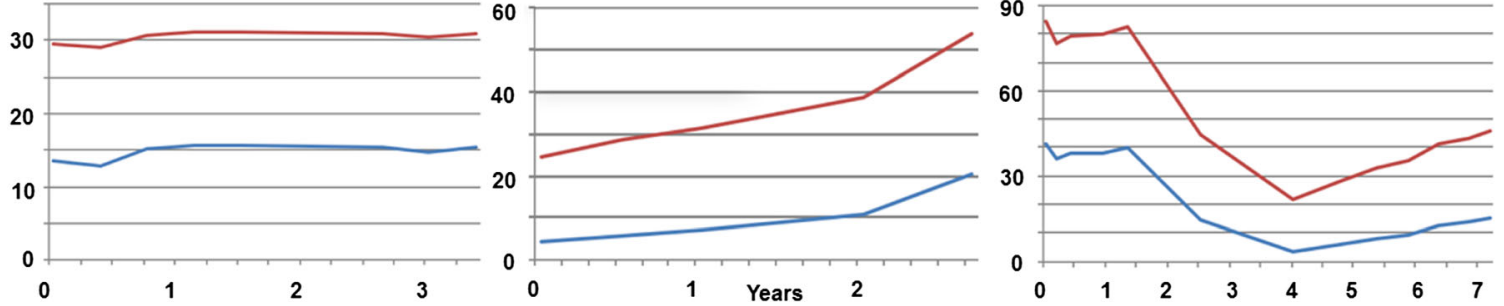

- Diameter $(\mathrm{mm})$

Fig. 1 Patients with tumor volume and calculated mean diameter over time $(x$-axis $=$ years $)$. Left: no sign of growth over 3.5 years. Middle: initial growth rate of $5.5 \mathrm{~mm} /$ year with a change of growth rate after year 2 of $25 \mathrm{~mm} /$ year indicating malignant transformation. Right:

resected at year 1.5 and demonstrated reduction of the volume of signal changes over time and then regrowth from year 4 with a yearly growth rate of $8 \mathrm{~mm} /$ year. All these where reported as no growth based on standard radiological evaluation
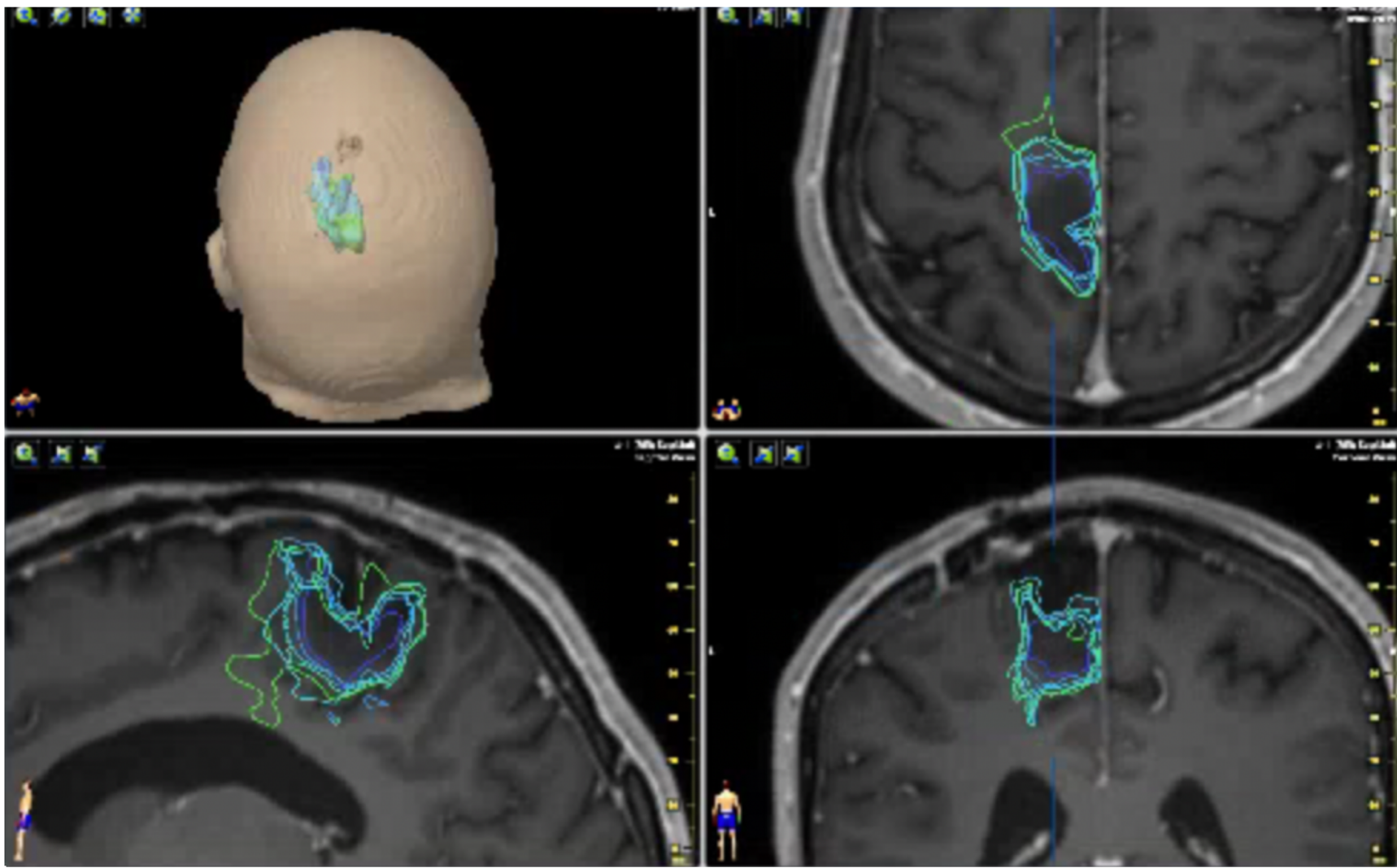

Fig. 2 A patient with a diffuse astrocytoma with FLAIR segmentations over 2.5 years (each time point in a separated color) overlaid on the latest $\mathrm{T} 1$ sequence for anatomical detail. It is seen that there progression in the

anterior direction and invasion of the corpus callosum. No growth was reported based on standard radiological evaluation

Publisher's note Springer Nature remains neutral with regard to jurisdictional claims in published maps and institutional affiliations.

\section{References}

1. Gui C, Lau JC, Kostenuik SE, Lee DH, Megyesi JF (2018) Radiology reporting of low-grade glioma growth underestimates tumour expansion. Acta Neurochir in press

2. Mandonnet E, Delattre JY, Tanguy ML, Swanson KR, Carpentier AF, Duffau H, Cornu P, Effenterre V, Alvord EC, Capelle L (2003) Continuous growth of mean tumor diameter in a subset of grade II gliomas. Ann Neurol 53(4):524-528 